\title{
Effects of shuangtengbitong tincture on collagen-induced arthritis in rats
}

\author{
WEI XU ${ }^{1}$, HUANG LI $^{1}$, KEDAN CHU $^{1}$, YUQIN ZHANG ${ }^{1}$, HAIYIN ZHENG $^{2}$, \\ MINGQING HUANG ${ }^{1}, \mathrm{XUN} \mathrm{ZHANG}^{1}$ and LIDIAN CHEN ${ }^{3}$ \\ ${ }^{1}$ Pharmacy College, ${ }^{2}$ Integrative Medicine College and ${ }^{3}$ Faculty of Rehabilitation Medicine, \\ Fujian University of Traditional Chinese Medicine, Fuzhou, Fujian 350122, P.R. China
}

Received March 12, 2013; Accepted September 9, 2013

DOI: $10.3892 / \mathrm{mmr} .2013 .1693$

\begin{abstract}
Shuangtengbitong tincture (STBT), a clinical prescription from the Fujian University of Traditional Chinese Medicine (TCM) Affiliated People's Hospital (Fuzhou, China), has been used in the treatment of rheumatoid arthritis (RA) for $\sim 10$ years. The aim of the current study was to confirm the anti-RA effect of STBT and to evaluate the potential mechanisms underlying collagen-induced arthritis (CIA) in rats. CIA model Wistar rats were induced with bovine type II collagen. The rats were immunized with CIA and were treated with STBT (0.5 and $2 \mathrm{ml} /$ injection) and votalin $(\sim 1 \mathrm{~cm} /$ injection $)$ continuously for $\sim 1$ month. Following treatment, the pathological sections of CIA rat joints were observed by hematoxylin and eosin staining, expression of toll-like receptor-4 (TLR4), myeloid differentiation factor $88(\mathrm{MyD} 88)$ and nuclear transcription factor- $\kappa \mathrm{B}$ $(\mathrm{NF}-\kappa \mathrm{B})$ were investigated by western blot analysis and reverse transcription-polymerase chain reaction (RT-PCR) analysis. Following treatment, STBT significantly suppressed paw swelling $(\mathrm{P}<0.05)$ compared with the model group and increased body weight. STBT also reversed pathological changes, STBT-treated rats showed a significant improvement of synovial hyperplasia, inflammatory infiltration, and cartilage and bone destruction. The levels of protein and mRNA expression of TLR4, MyD88 and NF- $\kappa$ B were markedly suppressed in the synovial tissue of STBT- and votalin-treated rats. In addition, STBT showed marked inhibition of the levels of protein and mRNA expression
\end{abstract}

Correspondence to: Professor Lidian Chen, Faculty of Rehabilitation Medicine, Fujian University of Traditional Chinese Medicine, 1 Huatuo Road, Minhou Shangjie, Fuzhou, Fujian 350122, P.R. China E-mail: clidianlab@gmail.com

Abbreviations: RA, rheumatoid arthritis; STBT, shuangtengbitong tincture; CIA, type II collagen-induced arthritis; TCM, traditional Chinese tedicines; HE, hematoxylin and eosin; TLR4, toll-like receptor-4; MyD88, myeloid differentiation factor 88; NF-кB, nuclear factor- $\kappa \mathrm{B}$

Key words: shuangtengbitong tincture, rheumatoid arthritis, toll-like receptor-4, MyD88, nuclear factor- $\kappa \mathrm{B}$ of TLR4, MyD88 and NF- $\mathrm{B}$ at an STBT volume ranging between 1 and $4 \mathrm{ml} / \mathrm{day}$, indicating that the inhibition was volume dependent. These results show that STBT inhibits CIA and may be correlated with TLR4, MyD88 and NF- $\kappa$ B expression.

\section{Introduction}

Rheumatoid arthritis (RA) is a systemic autoimmune disease, which is prevalent in $0.5-1 \%$ of the world's population (1). RA is chronic and may lead to joint damage, synovial membrane destruction, and cartilage and bone damage $(2,3)$. RA is an incurable disease that seriously impacts human physical and mental health (4). RA and the side effects of RA therapeutic agents may induce complications, including cardiovascular, communicable, blood, gastrointestinal and lung diseases (5). In addition, RA is accompanied by long duration, high treatment costs and high disability or mortality rate, which affects the quality of life of patients (6). At present, the etiology and pathogenesis of RA remains poorly understood; however, numerous pathways have been observed to contribute. The toll-like receptor (TLR) signal transduction pathway is considered to be an important pathway in the pathogenesis of RA. TLR family members are receptors involved in the immune system and microbial identification; they mediate the innate immune reaction and activate the acquired immune response. In addition, TLR4 has been shown to be important in autoimmune diseases, particularly in RA $(7,8)$. The correlation between TLR4 and nonbiological inflammatory injury has previously been shown (9-11). It is hypothesized that injured tissue and necrotic cells release endogenous activators, termed adjuvants. These activators bind to TLR4 on the cell membrane. MyD88, an adapter protein of TLR, is located in the cell and is the key signaling molecule in the signal transduction pathway. When cells are stimulated by an extracellular signal, nuclear transcription factor- $\kappa \mathrm{B}$ $(\mathrm{NF}-\kappa \mathrm{B})$ is activated, which promotes the transcription of a number of genes and the release of series a cytokines, resulting in inflammation (12-15) through the signaling cascade. Activated cells may also induce specific immunity to increase the self-protection ability of the organism. An excessive inflammatory reaction may also damage target cells and tissue. Thus, the effects of NF- $\kappa \mathrm{B}$ in the incidence 
and treatment of RA has been an area of interest in studies concerning rheumatism.

Traditional Chinese medicines (TCM) have been observed to be clinically effective and are the most prevalent treatment for RA in a number of Asian countries. RA is similar to $\mathrm{Bi}$ Zheng in TCM theory, which is defined as a characteristic syndrome of numbness and weakness, bone pain, joint stiffness and deformation, and dyskinesia (16). In TCM, it is generally considered that Bi Zheng occurs due to attack of the meridians of the limbs by exogenous wind, dampness and heat or cold pathogens $(16,17)$. Traditional Chinese herbal prescriptions have long been employed for the treatment of RA, pain-relief, anti-inflammation and/or as immunomodulatory therapies $(18,19)$. Traditional Chinese herbal prescriptions contain a number of herbs and collectively exert therapeutic and modulatory effects (20). These formulae have been demonstrated in numerous basic and clinical studies $(3,21,22)$.

STBT (Min drug system approval no. Z20111008; patent no. 201110347820.4) has been used as a clinical prescription for $\sim 10$ years and has become a standard hospital prescription at Fujian University of TCM Affiliated People's Hospital (Fuzhou, China). However, pharmacological studies have not yet been conducted and the underlying mechanisms of STBT remain to be fully elucidated. In the present study, the rat CIA model, a widely used experimental model of human RA, was used to investigate the effects of STBT on acute arthritis and chronic joint damage, and the potential mechanisms associated with the TLR signal transduction pathway.

\section{Materials and methods}

Materials and animals. A Dionex Ultimate 3000 liquid chromatography equipped with DAD detector was purchased from Dionex Ltd. (Sunnyvale, CA, USA); a KQ-500DE ultrasonic clearing machine was purchased from Kunshan Ultrasonic Instruments Co., Ltd. (Kunshan, China); a XS105 electronic analytical balance was obtained from Mettler-Toledo Instruments (Shanghai) Co., Ltd. (Shanghai, China); and the YLS-7B toe volume measuring instrument was purchased from Yi Yan Technology Development Co., Ltd. (Jinan, China).

Acetonitrile was of chromatographic grade and was used for high-performance liquid chromatography (HPLC). Reverse osmosis Milli-Q water (18 $\Omega$ M; Millipore, Bedford, MA, USA) was used throughout the study. All other chemical solvents in the study were at least of analytical reagent grade. Complete Freund's adjuvant (CFA) and type II collagen were purchased from Sigma-Alrdich (St. Louis, MO, USA). TRIzol reagent was purchased from Invitrogen Life Technologies (Carlsbad, CA, USA) and SuperScript II reverse transcriptase was provided by Promega Corporation (Madison, WI, USA). TLR4, MyD88 and NF- $\kappa \mathrm{B}$ primary antibodies and horseradish peroxidase (HRP)-conjugated secondary antibodies were purchased from Cell Signaling Technology Inc. (Beverly, MA, USA). All other chemicals used, unless otherwise stated, were obtained from commercial sources.

Specific pathogen-free (SPF) Wistar male rats $(n=50$; weight, 180-220 g) were provided by the Animal Care and Use Committee of Fujian University of TCM and were purchased from Shanghai Slac Laboratory Animal Co., Ltd. (Shanghai,

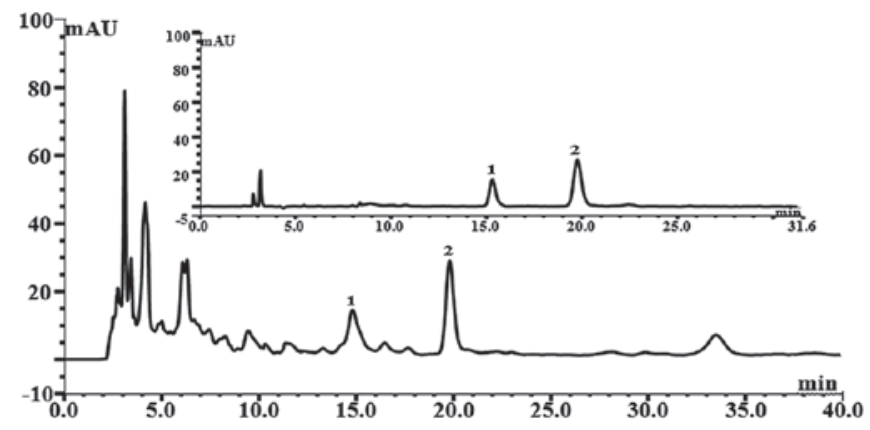

Figure 1. HPLC profile of target analytes in the STBT sample. Inset: HPLC profile of target analytes standard mixture. 1, triptolide; 2, sinomenine. HPLC, high-performance liquid chromatography; STBT, shuangtengbitong tincture.

China; license no. 200700518360). Animals were housed under controlled temperature $\left(21-23^{\circ} \mathrm{C}\right)$, relative humidity $55 \pm 5 \%$, and a 12-h light/dark cycle, and had access to standard rat chow and tap water ad libitum. All animal experiments were conducted in accordance with international Ethical Guidelines and the National Institutes of Health Guide concerning the Care and Use of Laboratory Animals.

\section{Methods}

Preparation of STBT. STBT is a mixture of four traditional drugs. The drugs were provided by Fujian University of TCM Affiliated People's Hospital and verified by Wei Lu (Pharmacy College of Fujian University of TCM). The voucher specimens were deposited from the Pharmacy College of Fujian University of TCM. STBT was prepared according to the preparation of the STBT hospital prescription of Fujian University of TCM Affiliated People's Hospital. Tripterygium wilfordii Hook.f., Sinomenium acutum Rehd. et Wils, Dioscorea ipponica Makino and Glycyrrhiza uralensis Fisch. were crushed into a coarse powder, mixed and steeped in $10 \mathrm{X} 80 \%$ ethanol. Following $48 \mathrm{~h}$ of slow percolation of the extract, the fluid from the percolation was collected and filtrated. Finally, the fluid was adjusted to $10 \mathrm{~g} / \mathrm{ml}$ (original medicinal materials/volume).

The prepared STBT was processed as previously described (23) and was filtrated through a $0.45 \mu \mathrm{m}$ microporous membrane and injected into the liquid chromatograph. Major peaks were identified as the marker compounds (Fig. 1).

CIA modeling and drug administration. Wistar rats $(\mathrm{n}=50)$ were randomly divided into five groups ( $\mathrm{n}=10$ per group); control; model; votalin ointment, an accepted control medicine clinically used to treat RA ( $\sim 1 \mathrm{~cm} /$ time$)$; STBT low-dose (0.5 $\mathrm{ml} /$ time) and STBT high-dose groups $(2 \mathrm{ml} /$ time $)$. Collagen $(2 \mathrm{mg} / \mathrm{ml})$ was emulsified with an equal volume of CFA to a final concentration of $0.1 \mathrm{mg} / \mathrm{ml}$. In addition to the control group, $0.2 \mathrm{mg}$ collagen emulsion was injected into the tail root of each rat by intradermal injection. Provocation testing was performed after 7 days using similar methods. Drugs were administered once daily 1 day following primary immunization. In the STBT low- and high-dose groups, STBT was sprayed onto the back of rats whose hair had been shaved twice daily. The area of the back where the drug was administered measured $3 \times 3 \mathrm{~cm}$. The votalin ointment group were 
administered votalin ointment in the same method as STBT. The control and model groups did not receive any treatment. All animals were treated for 35 days continuously.

Evaluation of paw swelling and body weight. A volume method was used to measure the paw volume of rats (24). The paw volume prior to modeling was measured initially and then 11 days following the primary immunization, the paw volume of each rat was measured every 4 days. Swelling was expressed by the volume difference $(\mathrm{ml})$ prior to and following modeling. The body weight of the rats was measured using an SE402F electronic scale [Ohaus Instruments (Shanghai) Co., Ltd., Shanghai, China] every 3 days from the primary immunization.

Histopathological examination. Following the termination of the experiment, rats were anesthetized and the right hind knee was removed and fixed in $4 \%$ neutral buffered formalin for 24 h. Following decalcification with $12.5 \%$ EDTA (pH 7.0) for 20 days, the right hind knee was paraffin-embedded, sectioned at a 5-mm thickness and stained with hematoxylin and eosin.

Western blot analysis. Synovial tissue was extracted from the hind paws of the rats and were homogenized with nondenaturing lysis buffer and centrifuged at $4^{\circ} \mathrm{C}$ at $12,000 \mathrm{x}$ g for $\sim 15 \mathrm{~min}$. The supernatants were denatured with protein loading buffer following the determination of the protein concentration. Protein $(45 \mu \mathrm{g})$ was resolved on $12 \%$ sodium dodecyl sulfate-polyacrylamide gel, blotted onto a polyvinylidene fluoride (PVDF) membrane and blocked for $2 \mathrm{~h}$ with $5 \%$ skimmed milk in Tris-buffered saline with Tween 20. Membranes were incubated with the desired primary antibody against TLR4, MyD88, NF- $\kappa \mathrm{B}$ or $\beta$-actin with a dilution of 1:1,000 overnight at $4^{\circ} \mathrm{C}$ and then with the appropriate HRP-conjugated secondary antibody for $50 \mathrm{~min}$. Following washing, the membranes were visualized by enhanced chemiluminescence detection.

RNA extraction and reverse transcription-polymerase chain reaction ( $R T-P C R)$ analysis. Total RNA was extracted from the synovial tissue of rats with TRIzol reagent. Total RNA $(2 \mu \mathrm{g})$ was reverse-transcribed to cDNA and used to determine the mRNA levels of TLR4, MyD88 and NF- $\kappa$ B by PCR with Taq DNA polymerase (Fermentas, Rockford, IL, USA). PCR was performed under the following conditions: Initial denaturation at $95^{\circ} \mathrm{C}$ for $3 \mathrm{~min}$, denaturation for $30 \mathrm{sec}$ at $95^{\circ} \mathrm{C}$, extension at $72^{\circ} \mathrm{C}$ for $45 \mathrm{sec}$ and amplification for 35 cycles. The annealing temperature was $62.8^{\circ} \mathrm{C}$ for TRL4, $65.5^{\circ} \mathrm{C}$ for MyD88 and $60^{\circ} \mathrm{C}$ for $\mathrm{NF}-\kappa \mathrm{B}$. Primers for TLR4, MyD88 and NF-кB were as follows: Forward: 5'-ATGCCAGGATGATGCCTCTCTTGCA-3' and reverse: 5'-TTCACACCTGGATAAATCCAGCCAC-3' for TLR4; forward: 5'-AGTTGCTAGCCTTGTTAGACC GTGAGG-3' andreverse: 5'-AAACAACCACCACCATGCGACGACACC-3' forMyD88; forward:5'-GCGCATCCAGACCAACAATAAC-3' and reverse: 5'-GCCGAA GCTGCATGGACACT-3' for NF- $\kappa$; ; and forward, 5'-GTCATCCATGACAACTTTGG-3' and reverse, 5'-GAGCTTGACAAAGTGGTCGT-3' for glyceraldehyde 3-phosphate dehydrogenase (GAPDH). GAPDH served as an internal control. All experiments were performed according to the manufacturer's instructions. Samples were analyzed by gel electrophoresis (1.5\% agarose) and the DNA bands were examined in a Gel Documentation System (Model Gel Doc 2000; Bio-Rad, Hercules, CA, USA).

Statistical analysis. Results are expressed as the mean \pm SD. Analysis of variance was used to determine significant differences between groups using SPSS Software (SPSS Inc, Chicago, IL, USA). $\mathrm{P}<0.05$ and $\mathrm{P}<0.01$ were considered to indicate a statistically significant difference.

\section{Results}

Effect of STBT on symptoms of CIA. Paw and knee joint swelling was an external objective indicator for evaluating the severity of inflammation in the RA model. This was monitored by an independent examiner who did not have prior knowledge of the experimental groups. The changes of paw swelling and body weight are presented in Tables I and II. Approximately three days after the second immunization, the rat knee joints began to swell and over time, the joint and paw size increased. Paw and knee joint volume increased and reached a maximum on $\sim$ day 18 . The groups receiving votalin ointment and high-dose STBT showed significant inhibition $(\mathrm{P}<0.01)$, compared with the model group. The body weight of the control group linearly increased, but the model group only increased 13 days prior to the generation of swelling and then decreased, particularly between the 13th and 16th day.

Effects of STBT on histopathological changes. Representative histopathological lesions in the hind knee joint of control, model, votalin ointment, STBT low- and high-dose groups are shown in Fig. 2. Synovium hyperplasia, disorganized arrangement, infiltration of inflammatory cells, cartilage destruction, bone destruction, a number of small blood vessels and pannus formation were observed in the model group. Histopatholgical changes were ameliorated in the treated groups to a different extent. The STBT high-dose group showed a significant improvement. This group exhibited only mild synovium proliferation, regular order of the cell morphology, infiltration of a small number of inflammatory cells, no typical pannus formation, the surface of the cartilage was smooth and there was no marked damage in cartilage and bone erosion.

Effect of STBT on the protein expression of TLR4, MyD88 and $N F-\kappa B$. As shown in Fig. 3, the synovial level of TLR4, MyD88 and NF- $\kappa$ B protein expression significantly increased in the model compared with the control group. Compared with the model group, treatment with STBT decreased the synovial levels of TLR4, MyD88 and NF- $\kappa$ B protein expression, particularly in the high-dose STBT group. The STBT high-dose group showed significant downregulation of TLR4, MyD88 and $\mathrm{NF}-\kappa \mathrm{B}$ protein expression $(\mathrm{P}<0.01)$ and the $\mathrm{STBT}$ low-dose group exhibited no marked effect on protein expression.

Effect of STBT on the levels of TLR4, MyD88 and NF- $\mathrm{BB}$ $m R N A$. The results of the RT-PCR assay (Fig. 4) showed that 

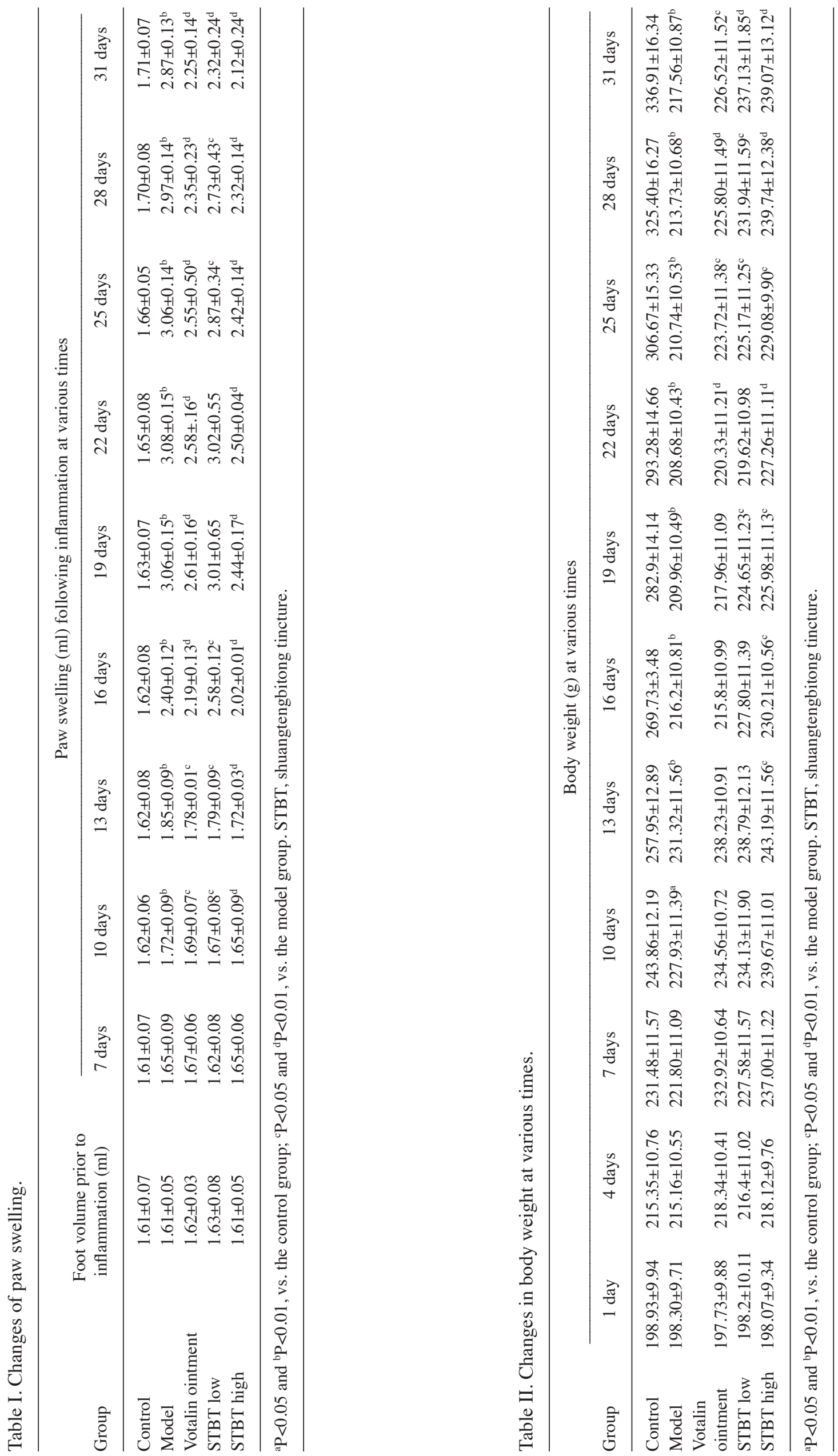
Table III. Composition of STBT.

\begin{tabular}{|c|c|c|c|c|}
\hline Pharmaceutical name & $\begin{array}{c}\text { Botanical } \\
\text { source/genus }\end{array}$ & Part used & Traditional actions/uses & Quantity (g) \\
\hline Tripterygium wilfordii Hook.f. & Tripterygium & Root & $\begin{array}{l}\text { Dispels wind and eliminate dampness; } \\
\text { dredges collaterals and relieves pain; } \\
\text { reduces swelling and eases pain }\end{array}$ & 30 \\
\hline Sinomenium acutum Rehd. et Wils & Sinomenium & Rattan & $\begin{array}{l}\text { Removes wind-dampness and } \\
\text { relieves pain, passes the meridian } \\
\text { and increases urination }\end{array}$ & 25 \\
\hline Dioscorea nipponica Makino & Dioscorea & Root and rhizome & $\begin{array}{l}\text { Dispels wind and eliminates } \\
\text { dampness; dredges collaterals and } \\
\text { passes the meridian; promotes blood } \\
\text { circulation and relieves pain }\end{array}$ & 25 \\
\hline Glycyrrhiza uralensis Fisch. & Glycyrrhiza & Root and rhizome & $\begin{array}{l}\text { Nourish Qi, alleviates pain, eliminates } \\
\text { phlegm, stops coughing, } \\
\text { regulates temperature and detoxifies }\end{array}$ & 15 \\
\hline
\end{tabular}

STBT, shuangtengbitong tincture.

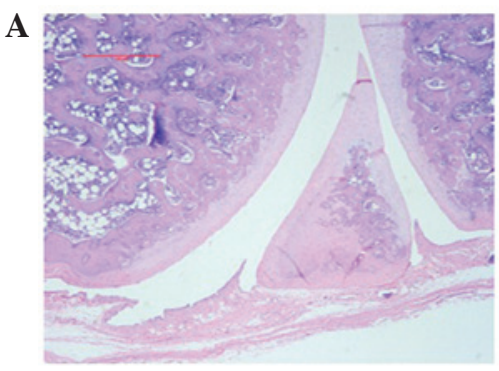

D

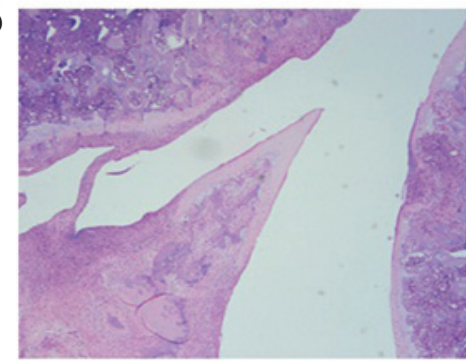

B

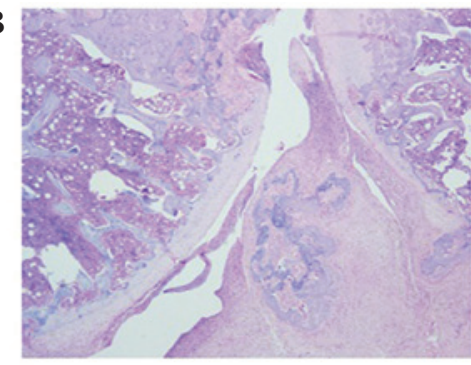

C

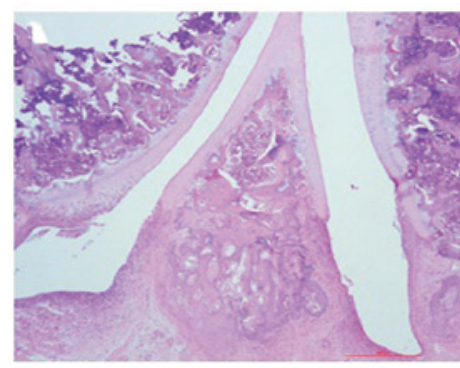

$\mathbf{E}$

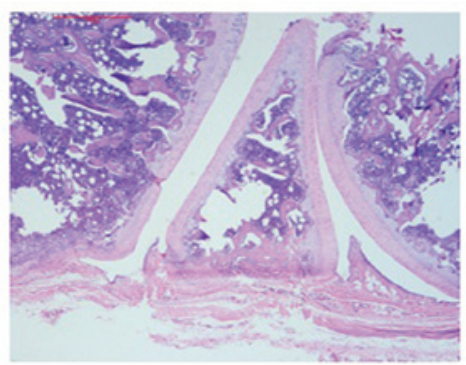

Figure 2. Effect of STBT on histological changes in CIA rats. Representative lesions using hematoxylin and eosin staining of rat hind knee joints were shown. (A) Control group; (B) model group; (C) votalin ointment group; (D) STBT low-dose group and (E) STBT high-dose group. STBT, shuangtengbitong tincture; CIA, collagen-induced arthritis.

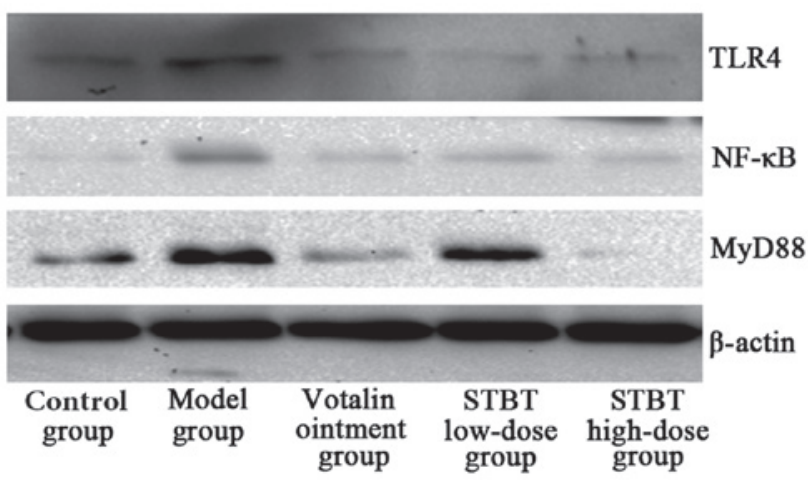

Figure 3. Effect of STBT on the protein expression of TLR4, MyD88 and

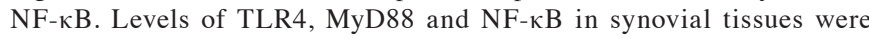
determined by western blot analysis. $\beta$-actin was used as the internal control. STBT, shuangtengbitong tincture; TLR4, toll-like receptor-4; MyD88, myeloid differentiation factor 88 ; NF- $\mathrm{\kappa B}$, nuclear transcription factor- $\kappa \mathrm{B}$.

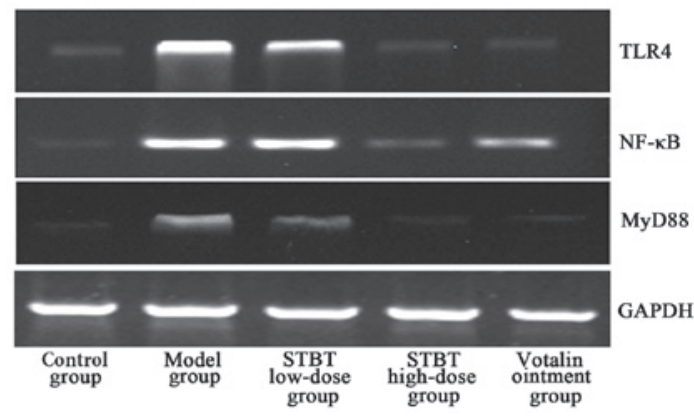

Figure 4. Effect of STBT on the mRNA expression of TLR4, MyD88 and NF-kB. Levels of TLR4, MyD88 and NF- $\mathrm{BB}$ in synovial tissues were determined by RT-PCR analysis. GAPDH was used as the internal control. STBT, shuangtengbitong tincture; TLR4, toll-like receptor-4; MyD88, myeloid differentiation factor 88 ; NF- $\mathrm{kB}$, nuclear transcription factor- $\mathrm{\kappa B}$; RT-PCR, reverse transcription-polymerase chain reaction. GAPDH, glyceraldehyde 3-phosphate dehydrogenase. 
the mRNA levels of TLR4, MyD88 and NF- $\mathrm{KB}$ in the model group was significantly increased compared with those in the control group $(\mathrm{P}<0.05)$. However, the mRNA levels were decreased following treatment with votalin ointment or various doses of STBT compared with the model group.

\section{Discussion}

STBT consists of four components, Tripterygium wilfordii Hook.f., Sinomenium acutum Rehd. et Wils, Dioscorea nipponica Makino and Glycyrrhiza uralensis Fisch (Table III). In STBT, the officinal part of Tripterygium wilfordii Hook.f. is the root. Its TCM properties are that it is bitter and acrid in taste, cool in nature and extremely toxic. It is associated with the liver and kidney channels. Its efficacies are dispeling wind and eliminating dampness, dredging collaterals, relieving pain and reducing swelling (25). The root of Tripterygium wilfordii Hook.f. is one of the most effective TCMs for the treatment of RA. Long-term clinical practice has shown that using Tripterygium wilfordii Hook.f. to treat RA may reduce or replace corticosteroids and/or steroidal anti-inflammatory drugs and possesses the advantages of high efficiency and low toxicity (26). It has been demonstrated that the predominant active ingredient, triptolide, is effective on the expression and activity of NF-KB in the synovium of CIA rats (26). Sinomenium acutum Rehd. et Wils is used in a number of TCM treatments, including removing wind-dampness and relieving pains, passing the meridian and increasing urination and it is used to treat pain and numbness in arthritis, swollen joints, paralysis and itching (27). Its predominate active ingredient, sinomenine, was used in the therapy of the RA and exhibited clear and definite therapeutic effects $(28,29)$. These include relaxing tendons, removing phlegm, removing wind-dampness and relieving pains. Dioscorea nipponica Makino is used to treat pain and numbness in arthritis, paralysis, Kashin-Beck disease, traumatic injury and bronchitis (30). It was employed in a number of formulae to clinically treat RA (16,31). Glycyrrhiza uralensis Fisch. nourishes Qi, alleviates pain, eliminates phlegm, stops coughing, regulates heat and detoxifies. Each component of STBT may be important in the treatment of RA. However, in TCM, a number of herbs are often combined under the theories of TCM. The combined interactions of these herbs are hypothesized to contribute more to the anti-RA effect of STBT compared with single use.

CIA is one of the most widely used experimental arthritis animal models for the identification of genes and mechanisms, and shares specific immunological and pathological features with human RA $(32,33)$. In the current study, it was observed that the swelling dimensions following treatment with STBT were decreased compared with the model group. According to the histopathological change of ankle joint analysis, it was hypothesized that STBT may alleviate the hyperplasia of the synovial membrane and bone destruction. STBT is hypothesized as a potential therapeutic agent for treating RA patients.

TLR signal transduction pathways are important in the inflammatory responses of RA. There are two primary pathways, the MyD88-dependent and MyD88-independent pathways. There are a number of adaptor proteins in the
TLR signal transduction pathway. MyD88 is a protein with a Toll-interleukin receptor structure domain and is significant in the effect of the TLR signal transduction pathway. MyD88 identifies pathogen-associated molecular patterns intra-articularly, magnifies the inflammation signal and forms a cascade reaction, resulting in the activation of the NF- $\mathrm{NB}$ signaling pathways. The inflammatory response is initiated and cells synthesize and release a number of inflammatory factors, including IL-1 $\beta$ and TNF- $\alpha$. TNF- $\alpha$ provides positive feedback and activates NF- $\kappa$ B. These activities effect the inflammatory factors and maintain the inflammation, which destroys the bone tissue. NF- $\mathrm{KB}$ was confirmed to modulate the ectopic expression of the FLICE inhibitory protein in RA synovial fibroblasts, inhibiting the apoptosis of synovial membrane fibroblasts (34). Inhibition of the NF- $\kappa \mathrm{B}$ signal pathway results in the downregulation of the expression of specific anti-apoptosis and cytokine genes (35).

In the current study TLR4, NF- $\mathrm{kB}$ and MyD88 in synovial tissue were determined by western blot analysis and RT-PCR. The expression level of NF- $\mathrm{KB}$ was consistent with previous studies (3,27-29) and TLR4 and MyD88 were downregulated. This may suggest that TLR4 and NF- $\mathrm{KB}$ signal transduction pathways are important in the RA inflammatory response.

In conclusion, the current results suggest that STBT is effective for the treatment of RA and modulates the inflammatory response, potentially by downregulating TLR4, NF- $\mathrm{KB}$ and MyD88. However, further studies are required to determine the pharmacological effects and molecular mechanisms of STBT.

\section{Acknowledgements}

This study wassupported by grants from theNational Technology Support Project of China (grant no. 2011BAI01B05); the major projects of Science and Technology Bureau of Fujian Province (grant no. 2009YZ0001-1-1); the major project of Fujian Provincial Health Bureau (grant no. WZZY0902); and the Fujian Province Science-Technology Plan Projects (grant no. 2010Y2004).

\section{References}

1. Brenner M, Meng HC, Yarlett NC, Joe B, Griffiths MM, Remmers EF, Wilder RL and Gulko PS: The non-MHC quantitative trait locus Cia5 contains three major arthritis genes that differentially regulate disease severity, pannus formation, and joint damage in collagen- and pristane-induced arthritis. J Immunol 174: 7894-7903, 2005.

2. Helliwell PS, Woodburn J, Redmond AC, Turner DE and Davys HJ (eds): Current concepts in rheumatoid arthritis. In: The Foot and Ankle in Rheumatoid Arthritis: A Compehensive Guide. Churchill Livingstone, Edinburgh, pp.1-16, 2007.

3. Yang M, Xiao C, Wu Q, Niu M, Yao Q, Li K, Chen Y, Shi C, Chen D, Feng G and Xia C: Anti-inflammatory effect of Sanshuibaihu decoction may be associated with nuclear factor-kappa B and p38 MAPK alpha in collagen-induced arthritis in rat. J Ethnopharmacol 127: 264-273, 2010.

4. Wolfe F, Michaud K, Gefeller O and Choi HK: Predicting mortality in patients with rheumatold arthritis. Arthritis Rheum 48: 1530-1542, 2003.

5. Tobón GJ, Youinou P and Saraux A: The environment, geo-epidemiology, and autoimmune disease: Rheumatoid arthritis. Autoimmun Rev 9: A288-A292, 2010.

6. Rat AC and Boissier MC: Rheumatoid arthritis: direct and indirecteosts. Joint Bone Spine 71: 518-524, 2004.

7. Cook DN, Hollingsworth JW Jr and Schwartz DA: Toll-like receptors and the genetics of innate immunity. Curr Opin Allergy Clin Immunol 3: 523-529, 2003. 
8. Migita K, Miyashita T, Maeda Y, Nakamura M, Yatsuhashi H, Kimura $\mathrm{H}$, Ishibashi $\mathrm{H}$ and Eguchi $\mathrm{K}$ : Toll-like receptor expression in lupus peripheral blood mononuclear cells. J Rheumatol 34: 493-500, 2007.

9. Zhang X, Li H, Hu S, Zhang L, Liu C, Zhu C, Liu R and Li C: Brain edema after intracerebral hemorrhage in rats: the role of inflammation. Neurol India 54: 402-407, 2006.

10. Li C, Ha T, Kelley J, Gao X, Qiu Y, Kao RL, Browder W and Williams DL: Modulating Toll-like receptor mediated signaling by $(1 \rightarrow 3)$-beta-D-glucan rapidly induces cardioprotection. Cardiovasc Res 61: 538-547, 2004.

11. Akira S, Uematsu S and Takeuchi O: Pathogen recognition and innate immunity. Cell 124: 783-801, 2006.

12. Wang C, Deng L, Hong M, Akkaraju GR, Inoue J and Chen ZJ: TAK1 is a ubiquitin-dependent kinase of MKK and IKK Nature 412: 346-351, 2001.

13. Sun L, Deng L, Ea CK, Xia ZP and Chen ZJ: The TRAF6 ubiquitin ligase and TAK1 kinase mediate IKK activation by BCL10 and MALT1 in T lymphocytes. Mol Cell 14: 289-301, 2004.

14. Qian Y, Commane M, Ninomiya-Tsuji J, Matsumoto K and Li X: IRAK-mediated translocation of TRAF6 and TAB2 in the interleukin-1-induced activation of NFkappa B. J Biol Chem 276 41661-41667, 2001.

15. Jiang L, Ninomiya-Tsuji J, Qian Y, Mastsumoto K and Li X: Interleukin-1 (IL-1) receptor-associated kinase-dependent IL-1-induced signaling complexes phosphorlate TAK1 and TAB2 at the plasma membrane and activate TAK1 in the cytosol. Mol Cell Biol 22: 7158-7167, 2002.

16. Lam FF, Ko IW, Ng ES, Tam LS, Leung PC and Li EK: Analgesic and anti-arthritic effects of Lingzhi and San Miao San supplementation in a rat model of arthritis induced by Freund's complete adjuvant. J Ethnopharmacol 120: 44-50, 2008.

17. Chen L: Bizheng of TCM and sub clinical. J Chin Med 3: 116, 2011 (In Chinese).

18. Deng ZZ, He YT and Wang ZQ: Research on the anti-inflammatory and analgesia effects of fufang leigongteng film on rheumatoid arthritis. Chinese Journal of Hospital 13: 14-16, 1998 (In Chinese)

19. Liang GX and Duan JM: Clinical effect observation of Silongsanmiao Powder on 68 rheumatoid arthritis cases. CJGMTCM 22: 86-87, 2007 (In Chinese).

20. Wang JF, Cai CZ, Kong CY, Cao ZW and Chen YZ: A computer method for validating traditional Chinese medicine herbal prescriptions. Am J Chin Med 33: 281-297, 2005.

21. Chang SH, Sung HC, Choi Y, Ko SY, Lee BE, Baek DH, Kim SW and Kim JK: Suppressive effect of AIF, a water extract from three herbs, on collagen-induced arthritis in mice. Int Immunopharmacol 5: 1365-1372, 2005.
22. Setty AR and Sigal LH: Herbal medications commonly used in the practice of rheumatology: mechanisms of action, efficacy, and side effects. Semin Arthritis Rheum 34: 773-784, 2005.

23. Li H, Xu W, Chu K, Chen L and Zhang X: Determination of triptolide in Bitongling tincture by HPLC. Chin J Inform Trad Chin Med 19: 51-52, 2012 (In Chinese).

24. Ganesan K, Tiwari M, Balachandran C, Manohar B and Puvanakrishnan R: Estrogen and testosterone attenuate extracellular matrix loss in collagen-induced arthritis in rats. Calcif Tissue Int 83: 354-364, 2008.

25. Liu P and Hu Y: Re-understanding of Tripterygium wilfordii Hook.f. J Chin Integr Med Rheumatol 5: 169, 1996 (In Chinese).

26. Tu S, Hu Y, Zeng K, Zhang M, Lai X and Weishen Z: Effects of triptolide on the expression and activity of NF-kappaB in synovium of collagen-induced arthritis rats. J Huazhong Univ Sci Technolog Med Sci 25: 543-545, 2005.

27. National Pharmacopoeia Committee: Chinese Pharmacopoeia. 1st ed. TCM Science and Technology Publishing House, Beijing, pp. 181-182, 2010 (In Chinese).

28. Fang Y, Wang Y, Zhou X, Zhong B, Bai G and Zhang C: Effect and mechanism of sinomenine on the signal transduction of the synovial cell nuclear factor- $\kappa \mathrm{B}$ in rats with adjuvant arthritis. Chin J Clin Rehabil 9: 204-205, 2005 (In Chinese).

29. Fang Y,Zhang Y and Wang Y: Effects of sinomenine on svnovioctve apoptosis, cell cycle and change of bcl- 2 protein in patients with rheumatoid arthritis. Chinese Remedies \& Clinics 4: 261-264, 2008.

30. National Pharmacopoeia Committee: Chinese Pharmacopoeia. 1st ed. TCM Science and Technology Publishing House, Beijing, pp. 250-251, 2010 (In Chinese).

31. Liu Y: Clinical observation of rheumatoid arthritis treated with Sanxiao ymzi decoction combined with lefiunomide. J TCM Univ Hunan 31: 17-20, 2011 (In Chinese).

32. Brand DD, Kang AH and Rosloniec EF: Immunopathogenesis of collagen arthritis. Springer Semin Immunopathol 25: 3-18, 2003.

33. Einhorn TA, Buckwalter JA and Sheldon RS (eds): Orthopaedic Basic Science. People's Health Publishing House, Beijing, pp. 987-1204, 2001 (In Chinese).

34. Bai S, Liu H, Chen KH, Eksarko P, Perlman H, Moore TL and Pope RM: NF-kappaB-regulated expression of cellular FLIP protects rheumatoid arthritis synovial fibroblasts from tumor necrosis factor alpha-mediated apoptosis. Arthritis Rheum 50: 3844-3855, 2004.

35. Osorio Y Fortéa J, Bukulmez H, Petit-Teixeira E, Michou L, Pierlot C, Cailleau-Moindrault S, Lemaire I, Lasbleiz S, Alibert O, Quillet P, Bardin T, Prum B, Olson JM and Cornélis F: Dense genome-wide linkage analysis of rheumatoid arthritis, including covariates. Arthritis Rheum 50: 2757-2765, 2004. 